\title{
Cardiac medication use in patients with suspected ischaemia without obstructive coronary arteries: sex differences and psychological distress
}

\section{P. M. C. Mommersteeg (D) - J. Roeters van Lennep · J. Widdershoven}

Accepted: 25 March 2021 / Published online: 5 May 2021

(C) The Author(s) 2021

\begin{abstract}
Background Ischaemia without obstructive coronary arteries (INOCA) is more prevalent in women and associated with psychological distress. Pharmacological treatment goals are angina relief and cardiovascular risk management. The present study aims to examine sex differences in cardiac and non-cardiac medication use, as well as medication and sex differences related to consistent psychological distress in patients with suspected INOCA.

Design A TweeSteden mild stenosis observational cohort study in patients with suspected INOCA as detected by ischaemic reason for referral and non-obstructive arteries based on coronary angiography or computed tomography.

Methods Medication documented in the hospital records of 488 patients ( $53 \%$ women) was coded as angina relief medication, blood-pressure-lowering medication, antithrombotics, statins, and non-cardiac medication, using the Anatomical Therapeutic Chemical code. Depressive symptoms and anxiety were recoded as 'consistent distress' (above the cutoff score for depression and anxiety on validated questionnaires), 'inconsistent distress' (above the cut-off for depression or anxiety) or 'no distress' (below the cut-off).
\end{abstract}

\section{P. M. C. Mommersteeg $(\bowtie) \cdot$ J. Widdershoven} Department of Medical and Clinical Psychology, Centre of Research on Psychology in Somatic Diseases, Tilburg University, Tilburg, The Netherlands

P.M.C.Mommersteeg@tilburguniversity.edu

J. Roeters van Lennep

Department of Internal Medicine, Vascular Medicine, Erasmus Medical Centre, Rotterdam, The Netherlands

\section{J. Widdershoven}

Department of Cardiology, Elisabeth-TweeSteden Hospital, Tilburg, The Netherlands
Results No sex differences were observed in cardiac medication use. Women used anxiolytic benzodiazepines more often ( $12 \%$ vs $4 \%, p=0.002)$ compared to men. Consistent distress was more prevalent in women $(22 \%$ vs $15 \%, p=0.004)$ and was related to the use of more angiotensin-converting enzyme inhibitors/angiotensin receptor blockers and diuretics in women and to calcium antagonist use as well as lower adherence levels in men. Women who reported chest pain more often received angina relief medication and blood-pressure-lowering medication than men.

Conclusion No sex differences were observed in cardiac medication use in patients with suspected INOCA. Psychological distress may reflect hypertension and subsequent medication use in women, and experiencing chest pain and subsequent medication use in men.

Keywords Ischaemia without obstructive coronary arteries - Ischaemic heart disease - Sex differences . Medication use $\cdot$ Depression $\cdot$ Anxiety

\section{What's new?}

- No sex differences were found in cardiac medication use in patients with suspected ischaemia without obstructive coronary arteries.

- Consistent psychological distress was related to the use of more angiotensin-converting enzyme inhibitors/angiotensin receptor blockers as well as diuretics in women.

- Psychological distress was related to more calcium antagonist use, as well as to lower medication adherence in men. 


\section{Advertisement placed here.}

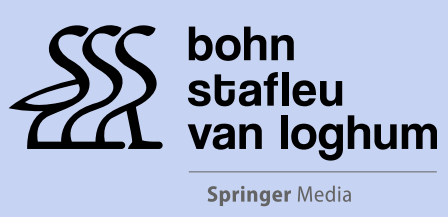

Houten 2021 


\section{Advertisement placed here.}

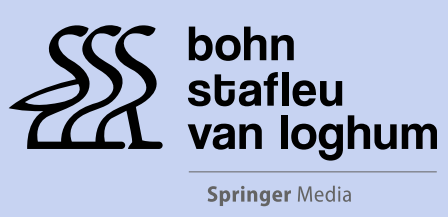

Houten 2021 


\section{Advertisement placed here.}

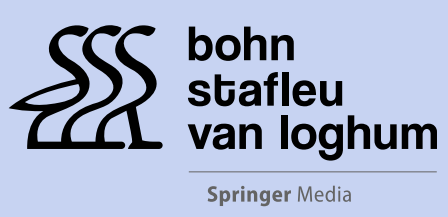

Houten 2021 


\section{Advertisement placed here.}

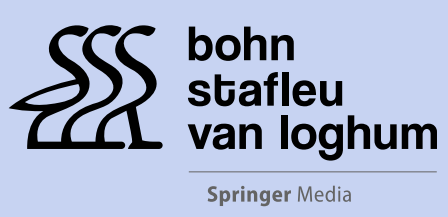

Houten 2021 


\section{Introduction}

Ischaemic heart disease (IHD) is the leading cause of death worldwide [1]. IHD without significant obstruction of the coronary arteries is referred to as non-obstructive coronary artery disease or, in the presence of signs and symptoms of ischaemia, ischaemia without obstructive coronary arteries (INOCA) [2]. Obstructive IHD is more prevalent in men, whereas INOCA is more prevalent in women [3]. Treatment for patients with IHD is based upon the European Society of Cardiology guidelines, consisting of managing cardiovascular risk factors by a healthy lifestyle and pharmacological therapy in combination with patient education $[4,5]$.

Psychosocial factors such as depression, anxiety and experiencing high levels of both anxiety and depressive symptoms indicating psychological distress are highly prevalent in IHD patients [6], particularly in women [7]. Psychological distress, depression, and anxiety are known to adversely affect medication adherence [8] and prognosis [9], for which appropriate referral is beneficial to reduce psychological symptoms $[6,10]$. In the present study, sex differences in the use of cardiac and non-cardiac medications and in psychological distress were examined in patients with suspected INOCA.

In patients with IHD, pharmacological therapy aims to provide relief for angina-like symptoms and to prevent future cardiac events $[4,5]$. A study comparing medical treatment between women and men among >52,000 Dutch patients after a myocardial infarction showed that women were less likely to receive optimal medication treatment, as indicated by the presence of acetylsalicylic acid, P2Y12 inhibitor, statins, beta blockers and angiotensin-converting enzyme/angiotensin 2 inhibitors [11]. It is unclear if these differences are present in patients with suspected INOCA. Women and men display differences in pharmacodynamics and pharmacokinetics, and women more often report more side effects [12]. Moreover, women with INOCA were more likely to be readmitted than men [3]. Still, women diagnosed with INOCA experience a two-fold increased risk for obstructive coronary artery disease (CAD) in the next 5-8 years and a four-fold increased risk for (cardiovascular) hospitalisation [13]. However, the absence of a sex-sensitive risk for adverse outcomes has been observed [14], and mortality rates were found to be similar for women and men with INOCA $[15,16]$. It is unknown if medication use is different for women and men with INOCA, and if these differences are apparent in women and men who report psychological distress.

We aim to add to this framework by examining sex differences in the medical treatment of patients with suspected INOCA. Suspected INOCA is defined as having visible wall irregularities or mild stenosis based on consecutive routine coronary angiography (CAG) or computed tomography (CT) scans in patients referred for cardiac complaints suspicious of ischaemia.
We hypothesise that women receive cardiac medication, including angina relief medication, as well as preventive cardiovascular medication such as anticoagulants/antiplatelets, statins, or blood-pressure-lowering drugs, less often than men. Second, we aim to examine the sex-stratified effect of psychological 'consistent distress', defined as experiencing high levels of both anxiety and depressive symptoms, on medication use and medication adherence. Given a higher symptom burden in patients with depressive symptoms or anxiety, we hypothesise that consistent psychological distress is related to more medication treatment, but lower adherence, for which sex differences may be apparent.

\section{Materials and methods}

\section{Participants and procedure}

The present study is a secondary analysis of the TweeSteden Mild Stenosis (TWIST) study [17]. Consecutive patients undergoing CAG $(n=5638)$ or 64 slice CT scan $(n=852)$ were screened between January 2009 and February 2013 [17]. All patients received treatment as usual from their cardiologist. Inclusion criteria were a CT calcium score above 0 without undergoing CAG, or CAG-detected visible mild stenosis ( $<50 \%$ left main coronary artery; $<70 \%$ other arteries). Exclusion criteria were absence of visible irregularities $(\leq 20 \%)$, significant coronary stenosis or a history of cardiac events. Eligible patients $(n=883)$ received information about the study, and 547 patients (62\%) gave their signed informed consent. Data were collected within 3 months after CAG or CT scan, and questionnaires were sent and returned by postal mail. Hospital record information on the reason for referral for CAG or CT scan was categorised to examine ischaemic [ $n=209,38 \%$; (unstable) angina pectoris; acute coronary syndrome; ischaemia based on electrocardiography, ergometry, or myocardial perfusion imaging] and suspected ischaemic origins $(n=238$, $44 \%$; inconclusive ergometry or myocardial perfusion imaging test; atypical chest pain). Patients referred for having a high number of risk factors, a high familial risk or other non-ischaemic reasons were excluded from the present analysis $(n=99,18 \%)$, leaving 448 patients with suspected INOCA. The research protocol was approved by the Medical Ethics Committee (METC Brabant; NL22258.008.08) and registered as an observational cohort study at ClinicalTrials.gov (NCT01788241).

\section{Medication and adherence}

Pharmacological products listed in hospital records were recoded as present or absent according to the Anatomical Therapeutic Chemical (ATC) code [18]. Treatment for patients without obstructive CAD aims to provide relief for angina-like symptoms us- 
ing nitrate vasodilators, beta blockers or calcium antagonists, and to prevent future cardiac events by lowering blood pressure, taking antithrombotic medication and/or lipid-lowering drugs $[4,5]$. Vasodilators included nitrate vasodilators (ATC code: C01DAxx): either short acting (C01DA02) or long acting (C01DA08, C01DA14). Blood-pressure-lowering medication included angiotensin-converting enzyme inhibitor (ACEI)/angiotensin II receptor blocker (ARB) [C09xxxx; angiotensin-converting enzyme inhibitor or an angiotensin II receptor type 1 antagonist (angiotensin II receptor blocker)], beta blockers (C07xxxx; C07AAxx, C07ABxx, C07BBxx), calcium antagonists [C08xxxx; C08DAxx, C08Dxx, with subgroup diltiazem (Tildiem; C08DB01)] and diuretics (C03xxxx, C07BBxx, C09BAxx, C09DAxx). Blood pressure medication was any of the above blood-pressure-lowering medications. Antithrombotic drugs included anticoagulants and antiplatelet drugs. Platelet inhibitors were B01ACxx; acetylsalicylic acid, or dipyridamole (B01AC07, B01AC30) and subgroups acetylsalicylic acid (B01AC06 aspirin or ASA), P2Y12 inhibitors (B01AC04 clopidogrel, B01AC24 ticagrelor, or B01AC30), and vitamin $\mathrm{K}$ antagonists (coumarins B01AA07 or B01AA04). No new oral anticoagulants were reported. Lipid-lowering medications were statins (C10xxxx; C10AAxx, C10ABxx, C10AXxx, C10BAxx, C10AX09).

Psychotropic drugs were grouped into antidepressants (N06AAxx, N06ABxx,) and sleep or anxiolytic benzodiazepines (N05CFxx/NO5CDxx, N05BAxx). Other non-cardiac medication included medication for diabetes (A10xxxx), chronic obstructive pulmonary disease (R03xxxx, R06xxxx), thyroid disease (H03AA01, H03BB02), proton pump inhibitors (A02BCxx), and non-steroidal anti-inflammatory drugs (M01Axxx).

In the questionnaire at 12 and 24 months patients were asked how often in the past month they had adhered to their medication treatment as prescribed by their physician, which was recoded as 'always' or 'not always'.

\section{Psychological distress}

Depressive symptoms and anxiety were reported using validated questionnaires, including the Beck Depression Inventory (BDI) for depressive symptoms and the Hospital Anxiety and Depression Scale for anxiety and depressive symptoms (HADS). The BDI has 21 items scored on a scale of $0-3$, with a cut-off of $\geq 10$ for moderate/severe depression. The HADS consists of two 7-item scales measuring anxiety (HADSA) and depressive symptoms (HADS-D), with a score range of $0-21$ and a cut-off score $\geq 8$ for moderate/ severe symptoms. Scoring above the cut-off value on each questionnaire was coded as 'consistent distress'. Scoring above the cut-off on one or two questionnaires was coded as 'inconsistent distress', and 'no distress' was the absence of values above the cut-off.

\section{Cardiac and non-cardiac risk factors}

Sociodemographic factors included sex, age, having a partner, having at least college education and lifestyle risk factors: body mass index (BMI) and obesity (BMI $\geq 30$ ), smoking ('current smoker' versus 'former smoker' or 'never smoked') and physical activity (being physically 'active' versus 'inactive' or 'moderately active'). Cardiac risk factors were obtained from hospital records: first-degree familial heart disease before 60 years, hypertension, hypercholesterolaemia, diabetes, and comorbid conditions. Disease severity was coded as inclusion via CAG versus CT scan, since CAG is related to a more severe disease presentation [19]. The number of coronary arteries with wall irregularities was reported. Chest pain was coded as part of the modified version of the Seattle Angina questionnaire as having had any angina, chest pain or chest tightness in the past 4 weeks [17].

\section{Statistics}

Analyses were completed with SPSS version 24. Sexstratified and/or distress-stratified differences were examined using one-way ANOVA for continuous variables and Pearson chi-square tests for categorical variables. In cases with a low number per cell, Fisher's exact test was used. Effect size phi-coefficient $\left[\varphi=\operatorname{SQRT}\left(\chi^{2} / N\right)\right]$ was calculated, for which $\varphi 0.1=$ small, $\varphi 0.3=$ medium and $\varphi 0.5=$ large. For descriptive purposes the prevalence of having medication in each category for having a specific risk factor was displayed for women and men, using chi-square tests to examine sex differences.

\section{Results}

Consistent distress was more prevalent in women (22\%) compared to men $\left(15 \%, \chi^{2}=11.0, p=0.004\right.$, effect size $\varphi=0.16$ ) (Tab. 1). Moreover, women were older, less often had a partner, and received less college education than men. Of the patients with suspected INOCA, 67\% had antithrombotic medication, statins (60\%), blood-pressure-lowering therapy $(67 \%)$ or angina relief medication $(62 \%)$; there were no significant sex differences (Tab. 2). Women used benzodiazepines (12\%) and thyroid medication (15\%) more often than men ( $4 \%$ and $2 \%$ respectively), with small-medium effect sizes (Tab. 2, range $\varphi=0.15-0.22$ ). Medication adherence at 12 months was not different between women and men, whereas more women $(87 \%)$ than men $(77 \%)$ reported always being adherent at 24 months $\left(\chi^{2}=5.7, p=0.017, \varphi=0.11\right)$.

Tab. 3 shows that in women with consistent distress, significantly more ACEI/ARB were used (43\%) than by women with inconsistent distress (28\%), and no distress $(22 \%)(\varphi=0.17)$. A similar pattern was observed for the use of diuretics $(41 \%, 25 \%$, $18 \% ; \varphi=0.20$ ). In men, the consistent distress group 
Table 1 Descriptive factors of patients with suspected ischaemia without obstructive coronary arteries, stratified by sex

\begin{tabular}{|c|c|c|c|c|c|}
\hline & & Women $(53 \%, N=239)$ & Men $(47 \%, N=209)$ & & \\
\hline & $N$ & $\%(n)$ or mean $(S D)$ & $\%(n)$ or mean (SD) & $\chi^{2} / F$ & $p$-value \\
\hline \multicolumn{6}{|l|}{ Sociodemographic factors } \\
\hline Age (years) & 448 & $63.32(9.04)$ & $60.51(9.60)$ & 10.19 & 0.002 \\
\hline With partner & 427 & $73 \%(169)$ & $90 \%(178)$ & 19.85 & $<0.001$ \\
\hline College education or higher & 424 & $43 \%(98)$ & $71 \%(140)$ & 33.33 & $<0.001$ \\
\hline \multicolumn{6}{|l|}{ Lifestyle risk factors } \\
\hline BMI $\left(\mathrm{kg} / \mathrm{m}^{2}\right)$ & 440 & $27.90(4.66)$ & $27.35(3.44)$ & 1.92 & 0.166 \\
\hline Obesity (BMI $\geq 30$ ) & 440 & $29 \%(69)$ & $21 \%(43)$ & 3.84 & 0.050 \\
\hline Smoking & 446 & $18 \%(43)$ & $21 \%(43)$ & 0.48 & 0.487 \\
\hline Physically active & 427 & $67 \%(154)$ & $58 \%(114)$ & 3.75 & 0.053 \\
\hline \multicolumn{6}{|l|}{ Cardiac risk factors } \\
\hline Familial heart disease $<60$ years & 421 & $68 \%(154)$ & $59 \%(116)$ & 3.41 & 0.065 \\
\hline Hypertension & 444 & $85 \%(201)$ & $84 \%(174)$ & 0.05 & 0.827 \\
\hline Hypercholesterolaemia & 446 & $67 \%(159)$ & $70 \%(146)$ & 0.59 & 0.443 \\
\hline Diabetes mellitus & 445 & $14 \%(32)$ & $13 \%(26)$ & 0.10 & 0.754 \\
\hline \multicolumn{6}{|l|}{ Reason for CAG/CT referral } \\
\hline Ischaemic ${ }^{a}$ & 239 & $54 \%(128)$ & $53 \%(111)$ & 0.01 & 0.925 \\
\hline Suspected ischaemic $^{b}$ & 209 & $46 \%(111)$ & $47 \%(98)$ & & \\
\hline \multicolumn{6}{|l|}{ Disease severity } \\
\hline Diagnosis via CAG (vs CT scan) & 448 & $73 \%(175)$ & $76 \%(159)$ & 0.48 & 0.489 \\
\hline Calcium score (mean \% in CT group) & 114 & $70.63(19.34)$ & $57.00(19.14)$ & 14.05 & $<0.001$ \\
\hline Visible wall irregularities & 448 & & & & \\
\hline One vessel & 101 & $28 \%(68)$ & $16 \%(33)$ & 10.77 & 0.005 \\
\hline Two vessels & 233 & $49 \%(118)$ & $55 \%(115)$ & & \\
\hline Three or more vessels & 114 & $22 \%(53)$ & $29 \%(61)$ & & \\
\hline Chest pain in the past month & 428 & $45 \%(103)$ & $52 \%(103)$ & 2.23 & 0.135 \\
\hline \multicolumn{6}{|l|}{ Comorbid conditions } \\
\hline Peripheral artery disease & 445 & $5 \%(12)$ & $6 \%(13)$ & 0.29 & 0.588 \\
\hline History of TIA or stroke & 445 & $4 \%(9)$ & $4 \%(8)$ & 0.00 & 0.979 \\
\hline COPD & 445 & $18 \%(42)$ & $11 \%(23)$ & 3.94 & 0.047 \\
\hline Inflammatory condition & 445 & $11 \%(25)$ & $7 \%(15)$ & 1.51 & 0.219 \\
\hline Gastro-intestinal condition & 445 & $16 \%(38)$ & $13 \%(26)$ & 1.12 & 0.289 \\
\hline Thyroid condition & 445 & $18 \%(42)$ & $2 \%(5)$ & 27.52 & $<0.001$ \\
\hline Skeletomuscular conditions & 445 & $9 \%(22)$ & $10 \%(21)$ & 0.08 & 0.772 \\
\hline Psychiatric condition & 445 & $6 \%(15)$ & $3 \%(6)$ & 2.92 & 0.087 \\
\hline \multicolumn{6}{|l|}{ Psychological distress } \\
\hline BDI depressive symptoms & 426 & $10.76(7.39)$ & $8.71(7.51)$ & 8.09 & 0.005 \\
\hline High BDI score $\geq 10$ & 426 & $48 \%(111)$ & $32 \%(62)$ & 12.13 & $<0.001$ \\
\hline HADS depressive symptoms & 427 & $5.45(3.98)$ & $5.19(4.19)$ & 0.44 & 0.509 \\
\hline High HADS-D score $\geq 8$ & 427 & $29 \%(66)$ & $26 \%(52)$ & 0.35 & 0.556 \\
\hline HADS anxiety & 427 & $7.13(4.31)$ & $5.96(4.29)$ & 7.86 & 0.005 \\
\hline High HADS-A score $\geq 8$ & 427 & $45 \%(103)$ & $32 \%(63)$ & 7.74 & 0.005 \\
\hline \multicolumn{6}{|l|}{ Psychological distress ${ }^{\complement}$} \\
\hline Consistent distress (all scores high) & 428 & $22 \%(51)$ & $15 \%(30)$ & 11.01 & 0.004 \\
\hline Inconsistent distress & & $39 \%(89)$ & $30 \%(59)$ & & \\
\hline No distress (all scores low) & & $39 \%(90)$ & $55 \%$ (109) & & \\
\hline
\end{tabular}


Table 2 Cardiac and non-cardiac medication stratified by sex

\begin{tabular}{|c|c|c|c|c|}
\hline & Women $(N=237)$ & $\operatorname{Men}(N=207)$ & Test value & $p$-value \\
\hline & $\%(n)$ & $\%(n)$ & $\chi^{2}$ & $p$ \\
\hline \multicolumn{5}{|l|}{ Cardiac medication } \\
\hline Antithrombotic & $64 \%(152)$ & $70 \%(145)$ & 1.75 & 0.187 \\
\hline - Platelet inhibitors (B01AC) & $62 \%(148)$ & $70 \%(145)$ & 2.84 & 0.092 \\
\hline - Acetylsalicylic acid (B01AC06) & $59 \%(141)$ & $67 \%(139)$ & 2.78 & 0.095 \\
\hline - P2Y12 inhibitor (B01AC04, B01AC24) & $5 \%(12)$ & $4 \%(8)$ & 0.37 & 0.544 \\
\hline - Vitamin $\mathrm{K}$ antagonist (B01AA) ${ }^{\mathrm{a}}$ & $3 \%(7)$ & $3 \%(6)$ & 0.00 & 0.973 \\
\hline Statins (C10) & $57 \%(135)$ & $64 \%(133)$ & 2.45 & 0.117 \\
\hline Blood-pressure-lowering medication & $69 \%(163)$ & $65 \%(135)$ & 0.63 & 0.426 \\
\hline - ACEI/ARB (C09) & $29 \%(68)$ & $29 \%(60)$ & 0.01 & 0.946 \\
\hline - Beta blockers (C07) & $47 \%(112)$ & $47 \%(98)$ & 0.00 & 0.986 \\
\hline - Calcium antagonists (C08) & $19 \%(44)$ & $17 \%(36)$ & 0.10 & 0.748 \\
\hline - Diltiazem (C08DB01) & $7 \%(16)$ & $6 \%(12)$ & 0.17 & 0.680 \\
\hline - Diuretics (C03/C07BB/C09) & $25 \%(59)$ & $18 \%(37)$ & 3.21 & 0.073 \\
\hline \multicolumn{5}{|c|}{$\begin{array}{l}\text { Use of antithrombotics, statins, or blood-pressure- } \\
\text { lowering medication }\end{array}$} \\
\hline - None & $9 \%(22)$ & $11 \%(23)$ & 4.51 & 0.212 \\
\hline - One & $22 \%(53)$ & $15 \%(32)$ & & \\
\hline- Two & $38 \%(89)$ & $36 \%(75)$ & & \\
\hline - Three & $31 \%(73)$ & $37 \%(77)$ & & \\
\hline Nitrates (C01DA) & $18 \%(42)$ & $16 \%(34)$ & 0.13 & 0.718 \\
\hline - Short-acting nitrates (C01DA02) & $11 \%(27)$ & $14 \%(29)$ & 0.69 & 0.407 \\
\hline - Long-acting nitrates (C01DA08, C01DA14) & $11 \%(26)$ & $6 \%(13)$ & 3.03 & 0.082 \\
\hline Angina relief medication ${ }^{b}$ & $63 \%(149)$ & $60 \%(124)$ & 0.41 & 0.522 \\
\hline \multicolumn{5}{|l|}{ Non-cardiac medication } \\
\hline \multicolumn{5}{|l|}{ Psychotropic medication } \\
\hline - Antidepressant use (N06A) & $10 \%(24)$ & $6 \%(12)$ & 2.78 & 0.095 \\
\hline - Benzodiazepine use (N05) ${ }^{\mathrm{a}}$ & $12 \%(28)$ & $4 \%(8)$ & 9.37 & 0.002 \\
\hline \multicolumn{5}{|l|}{ Other medication } \\
\hline - Diabetes medication (A10) & $10 \%(24)$ & $11 \%(22)$ & 0.03 & 0.863 \\
\hline - COPD medication (R03) & $13 \%(31)$ & $12 \%(24)$ & 0.23 & 0.635 \\
\hline - NSAIDs (M01A) ${ }^{\mathrm{a}}$ & $8 \%(18)$ & $3 \%(7)$ & 3.69 & 0.055 \\
\hline - Proton pump-inhibitors (A02BC) & $32 \%(76)$ & $25 \%(52)$ & 2.60 & 0.107 \\
\hline - Thyroid medication (H03AA01, H03BB02) & $15 \%(35)$ & $2 \%(5)$ & 20.57 & $<0.001$ \\
\hline \multicolumn{5}{|l|}{ Medication adherence $(N=401)$} \\
\hline - Always adherent at 12 months (vs not always) & $83 \%(139)$ & $78 \%(124)$ & 1.42 & 0.234 \\
\hline - Always adherent at 24 months & $87 \%(150)$ & $77 \%(126)$ & 5.66 & 0.017 \\
\hline
\end{tabular}

more often used calcium antagonists (33\%, 19\%, $13 \% ; \varphi=0.18)$ and proton pump inhibitors $(43 \%, 17 \%$, $23 \% \% ; \varphi=0.20)$. Antidepressant and benzodiazepine use was more prevalent in the consistent distress group in both women and men (range $\varphi=0.19-0.37$ ). Distressed men were less often 'always adherent', but only at 12 months $(55 \%, 77 \%, 84 \% ; \varphi=0.19)$. An explorative analysis showed that women with consistent distress, as compared to inconsistent or no distress, had hypertension significantly more often $(67 \%, 47 \%$, $45 \%, \varphi=0.18$, data not shown), whereas men with consistent distress more often reported chest pain (83\%, 58\%, 40\% respectively, $\varphi=0.31$, not shown).

Sex differences were explored for certain risk factors in the presence of medication for angina relief, lowering blood pressure, statins, or antithrombotics, as depicted in Fig. 1. Women who reported chest pain had angina relief medication (75\%) and blood-pressure-lowering medication $(79 \%)$ more often than men with chest pain $(60 \%, 63 \%$ respectively). 
Table 3 Cardiac and non-cardiac medication use stratified by sex and distress

\begin{tabular}{|c|c|c|c|c|c|c|c|c|}
\hline & \multicolumn{4}{|c|}{ Women $N=229$ (54\%) } & \multicolumn{4}{|c|}{ Men N= $197(46 \%)$} \\
\hline & $\begin{array}{l}\text { Consistent dis- } \\
\text { tress }\end{array}$ & $\begin{array}{l}\text { Inconsistent } \\
\text { distress }\end{array}$ & No distress & Test & $\begin{array}{l}\text { Consistent dis- } \\
\text { tress }\end{array}$ & $\begin{array}{l}\text { Inconsistent dis- } \\
\text { tress }\end{array}$ & No distress & Test \\
\hline & $22 \%(51)$ & $39 \%(89)$ & $39 \%(89)$ & & $15 \%(30)$ & $30 \%(59)$ & $55 \%(108)$ & $11.05^{\star \star}$ \\
\hline \multicolumn{9}{|l|}{ Cardiac medication } \\
\hline Antithrombotic & $67 \%(34)$ & $60 \%(53)$ & $66 \%(59)$ & 1.12 & $63 \%(19)$ & $76 \%(45)$ & $69 \%(75)$ & 1.75 \\
\hline Platelet inhibitors (B01AC) & $67 \%(34)$ & $60 \%(53)$ & $58 \%(52)$ & 1.00 & $63 \%(19)$ & $76 \%(45)$ & $64 \%(69)$ & 2.95 \\
\hline Statins (C10) & $55 \%(28)$ & $56 \%(50)$ & $58 \%(52)$ & 0.19 & $67 \%(20)$ & $69 \%(41)$ & $64 \%(69)$ & 0.54 \\
\hline $\begin{array}{l}\text { Blood-pressure-lowering medica- } \\
\text { tion }\end{array}$ & $73 \%(37)$ & $67 \%(60)$ & $69 \%(61)$ & 0.41 & $73 \%(22)$ & $68 \%(40)$ & $64 \%(69)$ & 1.00 \\
\hline - ACEI/ARB (C09) & $43 \%(22)$ & $28 \%(25)$ & $22 \%(20)$ & $6.79^{\star}$ & $47 \%(14)$ & $27 \%(16)$ & $26 \%(28)$ & 5.08 \\
\hline - Beta blockers (C07) & $53 \%(27)$ & $45 \%(40)$ & $47 \%(42)$ & 0.84 & $43 \%(13)$ & $51 \%(30)$ & $47 \%(51)$ & 0.47 \\
\hline - Calcium antagonists (C08) & $24 \%(12)$ & $17 \%(15)$ & $18 \%(16)$ & 1.01 & $33 \%(10)$ & $19 \%(11)$ & $13 \%(14)$ & $6.71^{*}$ \\
\hline - Diuretics (C03/C07BB/C09) & $41 \%(21)$ & $25 \%(22)$ & $18 \%(16)$ & $9.21^{\star *}$ & $30 \%(9)$ & $19 \%(11)$ & $16 \%(17)$ & 3.13 \\
\hline \multicolumn{9}{|c|}{$\begin{array}{l}\text { Use of antithrombotics, statins or blood-pressure- } \\
\text { lowering medication }\end{array}$} \\
\hline - None & $10 \%(5)$ & $10 \%(9)$ & $9 \%(8)$ & 2.15 & $7 \%(2)$ & $7 \%(4)$ & $14 \%(15)$ & 4.67 \\
\hline - One & $22 \%(11)$ & $22 \%(20)$ & $22 \%(20)$ & & $17 \%(5)$ & $12 \%(7)$ & $15 \%(16)$ & \\
\hline- Two & $33 \%(17)$ & $42 \%(37)$ & $35 \%(31)$ & & $43 \%(13)$ & $42 \%(25)$ & $31 \%(34)$ & \\
\hline - Three & $35 \%(18)$ & $26 \%(23)$ & $34 \%(30)$ & & $33 \%(10)$ & $39 \%(23)$ & $40 \%(43)$ & \\
\hline Nitrates (C01DA) & $22 \%(11)$ & $12 \%(11)$ & $21 \%(19)$ & 3.05 & $17 \%(5)$ & $24 \%(14)$ & $12 \%(13)$ & 3.84 \\
\hline Angina relief medication $^{a}$ & $67 \%(34)$ & $64 \%(57)$ & $61 \%(54)$ & 0.53 & $67 \%(20)$ & $61 \%(36)$ & $58 \%(63)$ & 0.70 \\
\hline \multicolumn{9}{|l|}{ Psychotropic medication } \\
\hline - Antidepressant (N06A) & $18 \%(9)$ & $13 \%(12)$ & $3 \%(3)$ & $8.44^{*}$ & $27 \%(8)$ & $5 \%(3)$ & $1 \%(1)$ & $27.34^{\star \star \star}$ \\
\hline - Benzodiazepine (N05) & $25 \%(13)$ & $9 \%(8)$ & $7 \%(6)$ & $12.06^{\star \star}$ & $13 \%(4)$ & $2 \%(1)$ & $3 \%(3)$ & $7.93^{*}$ \\
\hline \multicolumn{9}{|l|}{ Other medication } \\
\hline - Diabetes medication (A10) & $16 \%(8)$ & $11 \%(10)$ & $6 \%(5)$ & 3.87 & $20 \%(6)$ & $10 \%(6)$ & $8 \%(9)$ & 3.38 \\
\hline - COPD medication (R03) & $22 \%(11)$ & $9 \%(8)$ & $13 \%(12)$ & 4.38 & $20 \%(6)$ & $14 \%(8)$ & $9 \%(10)$ & 2.68 \\
\hline - NSAIDs (M01A) & $6 \%(3)$ & $7 \%(6)$ & $9 \%(8)$ & 0.55 & $7 \%(2)$ & $5 \%(3)$ & $2 \%(2)$ & 2.17 \\
\hline - Proton pump inhibitors (A02BC) & $41 \%(21)$ & $36 \%(32)$ & $25 \%(22)$ & 4.67 & $43 \%(13)$ & $17 \%(10)$ & $23 \%(25)$ & $7.71^{*}$ \\
\hline $\begin{array}{l}\text { - Thyroid medication (H03AA01, } \\
\text { H03BB02) }\end{array}$ & $20 \%(10)$ & $16 \%(14)$ & $12 \%(11)$ & 1.34 & $0 \%(0)$ & $7 \%(4)$ & $1 \%(1)$ & $6.21^{*}$ \\
\hline \multicolumn{9}{|l|}{ Medication adherence } \\
\hline - Always adherent at 12 months & $79 \%(31)$ & $83 \%(52)$ & $85 \%(56)$ & 0.50 & $55 \%(12)$ & $77 \%(40)$ & $84 \%(72)$ & $8.57^{\star}$ \\
\hline - Always adherent at 24 months & $86 \%(32)$ & $88 \%(58)$ & $87 \%(60)$ & 0.05 & $68 \%(15)$ & $80 \%(40)$ & $78 \%(71)$ & 1.28 \\
\hline \multicolumn{9}{|c|}{$\begin{array}{l}\text { \% }(n) \text { are reported, with } \chi^{2} \text { test values } \\
\text { Distress is defined as the presence of high levels of depression and anxiety according to the cut-offs for the HADS-D, HADS-A, and BDI (consistent distress), or } \\
\text { scores above the cut-off on one or two questionnaires (inconsistent distress), versus no scores above the cut-off (no distress) } \\
A C E l \text { angiotensin-converting enzyme inhibitor, } A R B \text { angiotensin II receptor blocker, COPD chronic obstructive pulmonary disease, NSAIDs non-steroidal anti- } \\
\text { inflammatory drugs, } H A D S \text { Hospital Anxiety and Depression Scale, BDI Beck Depression Inventory } \\
{ }^{*} p<0.01,{ }^{\star \star} p<0.05,{ }^{* \star \star} p<0.001 \\
\text { aAngina relief medication was use of either nitrate vasodilators, beta blockers or calcium antagonists }\end{array}$} \\
\hline
\end{tabular}

\section{Discussion}

Based on the TWIST single-centre cohort study of patients with suspected INOCA, no significant sex differences were present in cardiac medication use. Previously observed sex differences were based on patients following myocardial infarction [11]. Patients in the TWIST study had no history of myocardial infarction, and subsequently the prevalence of cardiac medication use was lower overall. Herscovici and colleagues reviewed studies of patients with INOCA, showing large variability (2-59\%) in hypertension/ angina treatment and statin medication use [20]. In total $60 \%$ of the patients in the TWIST study with suspected INOCA received a combination of hypertension/angina therapy and statins, without sex differences. According to the 2019 ESC Guidelines pharmacological management of patients with stable CAD aims for relief of angina symptoms and to prevent the occurrence of cardiovascular events [4]. In total $62 \%$ of the patients received medication for angina relief, and $71 \%$ of the patients had either a blood-pressurelowering medication, and antithrombotic medication, or a statin, whereas only $10 \%$ of the patients had none. Whether pharmacological management goals were met was not reported upon, which is a limitation. However, medication use in patients with a certain risk factor is depicted in Fig. 1, showing that 
Prevalence of medication use in female and male patients with suspected INOCA

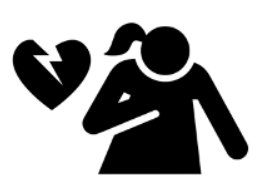

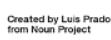

Prevalence of angina relief medication ${ }^{a}$

in patients (F/M) with... Chest pain: $\quad 75 \% / 60 \% *$ Hypertension: $80 \% / 81 \%$ Obesity: $\quad 76 \% / 77 \%$ Smoking: $\quad 51 \% / 70 \%$ Diabetes: $\quad 66 \% / 73 \%$

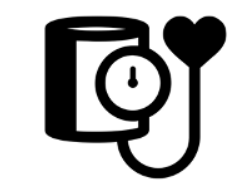

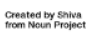

Prevalence of

blood pressure medication

in patients (F/M) with...

Chest pain: $\quad 79 \% / 63 \% *$

Hypertension: $93 \% / 93 \%$

Hyperchol.: $\quad 72 \% / 77 \%$

Obesity: $\quad 84 \% / 84 \%$

Smoking: $\quad 51 \% / 67 \%$

Diabetes: $\quad 78 \% / 81 \%$

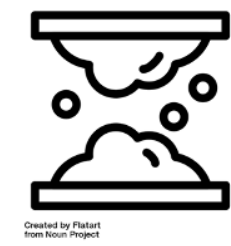

Prevalence of

in patients $(\mathrm{F} / \mathrm{M})$..

Included via CAG: $62 \% / 69 \%$

Hyperchol.: $79 \% / 83 \%$

Diabetes: $\quad 66 \% / 85 \%$

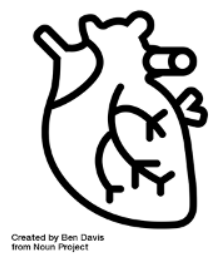

Prevalence of

antithrombotic medication ${ }^{c}$

in patients (F/M)..

Included via CAG: $\quad 70 \% / 77 \%$

INOCA: $\quad 67 \% / 75 \%$

Suspected INOCA: $60 \% / 65 \%$

Smoking: $\quad 77 \% / 67 \%$
Fig. 1 Sex-stratified prevalence of cardiac medication use in patients with suspected ischaemia without obstructive coronary arteries (INOCA). Hyperchol hypercholesterolaemia, CAG coronary angiography, ACEI angiotensin-converting enzyme inhibitors, $A R B$ angiotensin II receptor blockers.
aNitrate vasodilators, beta blockers, or calcium antagonists. bACEl/ARB-inhibitors, beta blockers, calcium antagonists, or diuretics. 'Acetylsalicylic acid, dipyridamole, P2Y12 inhibitors, or vitamin $\mathrm{K}$ antagonists. *Sex difference $p<0.05$ blood-pressure-lowering medication was received by $93 \%$ of patients with hypertension, and statins in $81 \%$ of the patients with hypercholesterolaemia.

Though women and men differ in pharmacokinetics and pharmacodynamics, which can affect the suggested dosage, effectiveness, and side effects [12, 21], current guidelines have no sex-specific recommendations for cardiac medication use, which may be reflected in the absence of sex differences in the present study.

Experiencing consistent distress was related to more blood-pressure-lowering ACEI/ARB and diuretic use in women, and to angina-relief calcium antagonist use in men. Explorative analysis subsequently showed significantly more hypertension in distressed women (but not men) and more chest pain in distressed men (but not women), which is in line with observed higher blood-pressure-lowering and antianginal medication use. A meta-analysis has reported psychosocial stress to be related to a higher prevalence and an increased risk of hypertension [22]. This may partially reflect an adverse lifestyle in people experiencing psychosocial stress, but can also reflect having an increased allostatic load of biological stress mechanisms associated with the development of hypertension [23]. Similarly, depressive symptoms and anxiety have been found to be associated with chest pain in previously published findings in the TWIST cohort, though without sex differences [17]. In addition to examining sex differences in medication use it remains relevant to further unravel mechanisms relating psychological distress to cardiovascular disease processes.

In line with other studies, psychological distress, including anxiety and depressive symptoms, was higher in women compared to men [9, 24]. More psychotropic medication use is observed in women compared to men in European countries [25], which is partially reflected in more benzodiazepine use in women in the present study, but no higher prevalence of antidepressant use.

At 24 months, but not 12 months, men showed a lower adherence compared to women, and distressed men showed a lower adherence than nondistressed men at 12 months, but not at 24 months. Whether this generic aspect of self-care behaviour could mediate an adverse outcome in men in the long term remains to be examined.

There are a number of limitations. No information was available regarding sex-specific factors such as hormone replacement therapy or menopause status [26]. The term sex instead of gender was used, though gender effects cannot be excluded. Medication use was based on hospital records, which may not reflect current medication use. Adherence was self-reported as a single item, with a severely skewed distribution; thus a more extensive questionnaire could be more reliable. We did not record the change in medication use based on the CAG or CT diagnosis, but reported the medication use within a few weeks to months after the index CAG or CT scan. No information on dosage was reported, and no information on side effects was requested. The present study is a single-centre observational cohort study, and exploring medication use was a secondary analysis. Whether these findings translate to other (Dutch) cardiology practices remains to be examined.

\section{Conclusion}

No sex differences were observed in cardiac medication use in patients with suspected INOCA, though consistent psychological distress in women reflected 
treatment for hypertension, and consistent distress in men reflected angina relief treatment.

Acknowledgements E. van den Munckhof coded the openended cardiac medication use with the corresponding ATC codes. T. Boll drafted a preliminary version of the manuscript, which has since been completely revised.

Conflict of interest P. M. C. Mommersteeg, J. Roetersvan Lennep and J. Widdershoven declare that they have no competing interests.

Open Access This article is licensed under a Creative Commons Attribution 4.0 International License, which permits use, sharing, adaptation, distribution and reproduction in any medium or format, as long as you give appropriate credit to the original author(s) and the source, provide a link to the Creative Commons licence, and indicate if changes were made. The images or other third party material in this article are included in the article's Creative Commons licence, unless indicated otherwise in a credit line to the material. If material is not included in the article's Creative Commons licence and your intended use is not permitted by statutory regulation or exceeds the permitted use, you will need to obtain permission directly from the copyright holder. To view a copy of this licence, visit http://creativecommons.org/licenses/by/4.0/.

\section{References}

1. Roth GA, Johnson C, Abajobir A, et al. Global, regional, and national burden of cardiovascular diseases for 10 causes, 1990 to 2015. JAm Coll Cardiol. 2017;70:1-25.

2. Kunadian V, Chieffo A, Camici PG, et al. An EAPCI expert consensus document on ischaemia with non-obstructive coronary arteries in collaboration with European Society of Cardiology Working Group on Coronary Pathophysiology \& Microcirculation endorsed by Coronary Vasomotor Disorders International Study Group. Eur Heart J. 2020;41:3504-20.

3. Bairey Merz CN, Pepine CJ, Walsh MN, FlegJL. Ischemia and no obstructive coronary artery disease (INOCA): developing evidence-based therapies and research agenda for the next decade. Circulation. 2017;135:1075-92.

4. Piepoli MF, Hoes AW, Agewall S, et al. 2016 European Guidelines on cardiovascular disease prevention in clinical practice. EurJ Prev Cardiol. 2016;23:Np1-NP96.

5. Knuuti J, Wijns W, Saraste A, et al. 2019 ESC guidelines for the diagnosis and management of chronic coronary syndromes. Eur Heart J. 2019;42:407-7.

6. Pedersen SS, von Känel R, Tully PJ, Denollet J. Psychosocial perspectives in cardiovascular disease. Eur J Prevent Cardiol. 2017;24(3_suppl):108-15.

7. Mommersteeg PMC, Widdershoven JWMG, Kop WJ. Sex and gender differences in psychosocial risk factors for ischemic heart disease. In: Maas AHEM, Merz CNB, editors. Manual of gynecardiology:female-specific cardiology. Cham: Springer;2017. pp. 203-20.

8. Bet PM, Penninx BW, van Laer SD, Hoogendijk WJ, Hugtenburg JG. Current and remitted depression and anxiety disorders as risk factors for medication nonadherence. J Clin Psychiatry. 2015;76:e1114-e21.

9. Vaccarino V, Badimon L, Bremner JD, et al. Depression and coronary heart disease: 2018 ESC position paper of the working group of coronary pathophysiology and microcirculation developed under the auspices of the ESC Committeefor Practice Guidelines. Eur Heart J. 2020;41:1687-96.
10. Richards SH, Anderson L, Jenkinson CE, et al. Psychological interventions for coronary heart disease. Cochrane Database Syst Rev. 2017;4:CD002902

11. Eindhoven DC, Hilt AD, Zwaan TC, Schalij MJ, Borleffs CJW. Age and gender differences in medical adherence after myocardial infarction: women do not receive optimal treatment-the Netherlands claims database. Eur J Prev Cardiol. 2018;25:181-9.

12. Oertelt-Prigione S, Regitz-Zagrosek V. Gender aspects in cardiovascular pharmacology. J Cardiovasc Transl Res. 2009;2:258-66.

13. Shaw LJ, Min JK, Narula J, et al. Sex differences in mortality associated with computed tomographic angiographic measurements of obstructive and nonobstructive coronary artery disease: an exploratory analysis. Circ Cardiovasc Imaging. 2010;3:473-81.

14. Bekendam MT, Kop WJ, Barzilay S, et al. The predictive value of positive affect and Type D personality for adverse cardiovascular clinical outcomes in patients with nonobstructive coronary artery disease. J Psychosom Res. 2018;104:108-14.

15. Pepine CJ, Ferdinand KC, Shaw LJ, et al. Emergence of nonobstructive coronary artery disease: a woman's problem and need for change in definition on angiography. JAm Coll Cardiol. 2015;66:1918-33.

16. Jespersen L, Hvelplund A, AbildstromSZ, etal. Stableangina pectoris with no obstructive coronary artery disease is associated with increased risks of major adverse cardiovascular events. Eur Heart J. 2012;33:734-44.

17. Mommersteeg PMC, Widdershoven JW, Aarnoudse W, Denollet J.Personality subtypes and chest pain in patients with nonobstructive coronary artery disease from the TweeSteden Mild Stenosis study: mediating effect of anxiety and depression. Eur J Pain. 2016;20:427-37.

18. World Health Organization. WHOCC - ATC/DDD Index 2019. 2019. https://www.whocc.no/atc_ddd_index/. Accessed: Feb 052020

19. Mommersteeg PMC, Arts L, Zijlstra W, et al. Impaired health status, psychological distress, and personality in women and men with nonobstructive coronary artery disease: sex and gender differences: the TWIST (Tweesteden Mild Stenosis) study. Circ Cardiovasc Qual Outcomes. 2017;10:1-9.

20. Herscovici R, Sedlak T, Wei J, et al. Ischemia and no obstructive coronary artery disease ( INOCA ): what is the risk? JAm Heart Assoc. 2018;7:e8868.

21. Rosano GM, Lewis B, Agewall S, et al. Gender differences in the effect of cardiovascular drugs: a position document of the Working Group on Pharmacology and Drug Therapy of the ESC. Eur Heart J. 2015;36:2677-80.

22. Liu MY, Li N, LiWA, Khan H. Association between psychosocial stress and hypertension: a systematic review and metaanalysis. Neurol Res. 2017;39:573-80.

23. Mocayar Marón FJ, Ferder L, Saraví FD, Manucha W. Hypertension linked to allostatic load: from psychosocial stress to inflammation and mitochondrial dysfunction. Stress. 2019;22:169-81.

24. Smaardijk VR, Maas AHEM, Lodder P, Kop WJ, Mommersteeg PMC. Sex and gender-stratified risks of psychological factors for adverse clinical outcomes in patients with ischemic heart disease: a systematic review and metaanalysis. Int JCardiol. 2020;302:21-9.

25. Boyd A, Van der Velde S, Pivette M, et al. Gender differences in psychotropic use across Europe: results from a large cross-sectional, population-based study. Eur Psychiatry. 2015;30:778-88.

26. Vaccarino V, Badimon L, Corti R, et al. Presentation, management, and outcomes of ischaemic heart disease in women. Nat Rev Cardiol. 2013;10:508-18. 